\title{
Design and High-Throughput Generation of Artificial Small RNA Constructs for Plants
}

\author{
Alberto Carbonell \\ Instituto de Biología Molecular y Celular de Plantas (Consejo Superior de Investigaciones \\ Cientificas-Universidad Politécnica de Valencia), 46022 Valencia, Spain
}

Running head: Generation of plant artificial sRNA constructs 


\begin{abstract}
Artificial microRNAs (amiRNAs) and synthetic trans-acting small interfering RNAs (syntasiRNAs) are two classes of 21-nucleotide artificial small RNAs (sRNAs) designed to selectively silence transcripts in plants with high efficacy and specificity. Despite their extensive use during the last decade, methods for designing and generating artificial sRNA constructs have not been optimized for time and cost-effectiveness and high-throughput applicability since recently. In this chapter, I detail the protocols for both the rationale design and high-throughput generation of plant artificial sRNA constructs using the P-SAMS ("Plant Small RNA Maker Suite") web tool and a new generation of $B s a \mathrm{I} / c c d \mathrm{~B}(\mathrm{~B} / \mathrm{c})$ vectors optimized for one-step cloning of artificial sRNA inserts. These protocols allow for the efficient generation of large number of amiRNA and syn-tasiRNA constructs for potent, selective and specific gene silencing in plants.
\end{abstract}

Key words: artificial small RNA, amiRNA, syn-tasiRNA, RNA silencing, P-SAMS 


\section{Introduction}

In plants, the relative simplicity and high efficacy of classic RNA interference (RNAi) approaches based on the expression of transgene-derived double-stranded RNAs (dsRNAs) explains their extensive use for selective gene silencing in gene function studies and crop improvement. However, the high risk of accidental targeting of cellular transcripts sharing high sequence complementarity with dsRNA-derived small interfering RNA (siRNA) species limits the specificity and, therefore, the application of this type of strategies. This important limitation has been recently overcome by the development of a new generation of highly specific RNAi technologies based on artificial small RNAs (sRNAs) [1].

Artificial sRNAs, including artificial microRNAs (amiRNAs) [2] and synthetic trans-acting small interfering RNAs (syn-tasiRNAs) [3-5], are 21-nucleotide (nt) RNAs designed to selectively silence transcripts in plants with high efficacy and specificity [1]. Both classes of artificial sRNAs exploit endogenous sRNA-directed silencing pathways for their biogenesis and function (Fig. 1). They are produced in planta by expressing a transgene including a functional miRNA or tasiRNA precursor with modified miRNA or tasiRNA sequences, respectively. While amiRNAs arise from DICER-LIKE1 (DCL1) cleavage of precursors with foldback structures, syn-tasiRNAs are generated after cleavage of a $T A S$ precursor by a miRNA/ARGONAUTE (AGO) complex, RNA-DEPENDENT RNA POLYMERASE 6 (RDR6) synthesis of dsRNA from one of the cleavage products, and DLC4-mediated processing of such dsRNA into 21-nt phased syn-tasiRNAs in register with the miRNA-guided cleavage site. Despite having different biogenesis pathways, both classes of artificial sRNAs associate with an AGO protein, usually AGO1, to target and silence highly sequence complementary transcripts (Fig. 1) [6]. AmiRNAs are particularly used to target single or sequence related genes, while syn-tasiRNAs can be used to target different sites in a single gene or multiple sequence-unrelated genes simultaneously [1] 
Despite both classes of artificial sRNAs have been widely used during the last decade, methods for designing and synthesizing artificial sRNAs have not been optimized for time and cost-effectiveness and high-throughput applicability since very recently [7-9]. The present chapter describes the methodology for the rationale design and high-throughput generation of large number of artificial sRNA constructs for plants using i) the P-SAMS ("Plant Small RNA Maker Suite") web tool [9] for the simple and fast automated design of artificial sRNAs through a user-friendly, modern interface and wizard assisted navigation which guides the user through the whole design process; and ii) a new generation of $B s a \mathrm{I} / c c d \mathrm{~B}(\mathrm{~B} / \mathrm{c})$ artificial sRNA vectors optimized for a zero-background, one-step cloning of artificial sRNA inserts resulting from the annealing of two overlapping and partially complementary oligonucleotides $[7,8]$. Specific protocols for the design and synthesis of both amiRNA and syn-tasiRNA constructs are provided. These protocols have been successfully applied very recently for the fast-forward identification of artificial sRNAs with high antiviral or antiviroid activity [10-12].

\section{Materials}

\subsection{Artificial sRNA Design}

1. Computer connected to the internet.

2. Web browser (e.g. Google Chrome, Safari, Mozilla Firefox, Internet Explorer).

\subsection{Artificial sRNA Cloning}

1. Oligo Annealing buffer: $60 \mathrm{mM}$ Tris- $\mathrm{HCl}$ (pH 7.5), $500 \mathrm{mM} \mathrm{NaCl}, 60 \mathrm{mM} \mathrm{MgCl} 2,10 \mathrm{mM}$ DTT (see Note 1).

2. Thermocycler or water bath.

3. Sterile $\mathrm{H}_{2} \mathrm{O}$.

4. T4 DNA ligase ( $5 \mathrm{U} / \mu \mathrm{L}$, Thermo Fisher Scientific). 
5. BsaI (10 U/ $\mu \mathrm{L}$, New England Biolabs).

6. B/c vectors $[7,8]$ (see Note 2). Each vector contains a unique combination of bacterial and plant antibiotic resistance genes.

7. Competent cells of Escherichia coli $c c d$ B-sensitive strain (e.g. DH5 $\alpha$, DH10B, TOP10).

8. LB agar plates with kanamycin: $20 \mathrm{~g} / \mathrm{L}$ of LB, $15 \mathrm{~g} / \mathrm{L}$ of Bacto Agar and $50 \mathrm{mg} / \mathrm{L}$ of kanamycin.

9. Liquid LB with kanamycin: $20 \mathrm{~g} / \mathrm{L}$ of $\mathrm{LB}$ and $50 \mathrm{mg} / \mathrm{L}$ of kanamycin.

10. GeneJET Plasmid Miniprep Kit (Thermo Fisher Scientific).

11. Oligonucleotides: M13-F (CCCAGTCACGACGTTGTAAAACGACGG), M13-R (CAGAGCTGCCAGGAAACAGCTATGACC), attB1 (ACAAGTTTGTACAAAAAAGCAGGCT) and attB2 (ACCACTTTGTACAAGAAAGCTGGGT).

12. Competent cells of Agrobacterium tumefaciens GV3101 strain.

\section{Methods}

The main steps regarding the design and synthesis of plant artificial sRNA constructs are summarized in Fig. 2. The whole process from artificial sRNA design to plant transformation is completed within a week.

\subsection{High-Throughput Generation of AmiRNA Constructs}

\subsubsection{AmiRNA Design}

P-SAMS amiRNA Designer web app [9] is used to design amiRNAs with an AGO1-preferred 5'U nucleotide, a C in position 19 to produce a star strand with an AGO1 non-preferred 5'G thus limiting the competition for AGO1 association with the guide strand, and with an intentional mismatch with the target transcript at position 21 to reduce transitivity. The app 
designs amiRNA to target one or multiples genes in different eudicot or monocot plant species (see Note 3). The app outputs the sequence of an amiRNA together with the sequence of the two oligonucleotides required for cloning the amiRNA in $\mathrm{B} / \mathrm{c}$ vectors containing the Arabidopsis thaliana MIR390a (Fig. 3a) or the Oryza sativa MIR390 (Fig. 4a) foldbacks for use in eudicot or monocot species, respectively (see Note 4). The protocol described next is intended for the design of amiRNAs targeting endogenous plant transcripts. The possibility of designing amiRNAs to target exogenous transcripts is also discussed.

1. Go to P-SAMS website (http://p-sams.carringtonlab.org/).

2. Click the "P-SAMS amiRNA Designer" application button to enter the amiRNA Designer tool (http://p-sams.carringtonlab.org/amirna/designer).

3. To start the design process, click "Get Started" and then "Design an amiRNA" (see Note 5).

4. Select the transcriptome of the species of interest and click "Yes" (see Notes 6 and 7).

5. Click "Option 1" if you have the gene ID(s) of the target transcript(s) (see Note 8).

6. Enter the gene ID(s) of the target transcript(s) and click "Next" (see Note 9).

7. Click "Yes" to have the results automatically filtered based on target specificity (see Note 10).

8. Click "Submit" to submit the job (see Note 11).

9. Select "Click to see Results" to display the results. AmiRNAs predicted to target uniquely desired target transcript(s) are output as "Optimal Results"; amiRNAs predicted to target desired target transcript(s) and endogenous transcripts are output as "Sub-optimal Results" (see Note 12).

10. Click "Download" to download the result summary including a list of amiRNA sequences together with the sequence of the two oligonucleotides required for cloning each amiRNA insert in compatible amiRNA B/c vectors (Fig. 3a and Fig. 4a) (see Note 13). 


\subsubsection{AmiRNA Cloning}

Diagrams of the main steps for the high-throughput cloning of amiRNA sequences in eudicot and monocot B/c vectors are shown in Fig. $\mathbf{3 b}$ and Fig. $\mathbf{4 b}$, respectively.

1. Resuspend the two oligonucleotides in sterile $\mathrm{H}_{2} \mathrm{O}$ to a final concentration of $100 \mu \mathrm{M}$.

2. Assemble the oligonucleotide annealing reaction in a PCR tube as described below:

\begin{tabular}{|l|l|}
\hline Forward oligonucleotide $(100 \mu \mathrm{M})$ & $2 \mu \mathrm{L}$ \\
\hline Reverse oligonucleotide $(100 \mu \mathrm{M})$ & $2 \mu \mathrm{L}$ \\
\hline Oligo Annealing buffer & $46 \mu \mathrm{L}$ \\
\hline
\end{tabular}

3. Transfer the tube to a thermocycler set to heat the annealing reaction $5 \mathrm{~min}$ at $94^{\circ} \mathrm{C}$ and then cool down to $20^{\circ} \mathrm{C}$ at a rate of $0.05^{\circ} \mathrm{C} / \mathrm{s}$ (see Note 14$)$.

4. Dilute the annealed oligonucleotides to a final concentration of $0.15 \mu \mathrm{M}$ as described below (see Note 15):

\begin{tabular}{|l|l|}
\hline Annealed oligonucleotides & $3 \mu \mathrm{L}$ \\
\hline $\mathrm{H}_{2} \mathrm{O}$ & $37 \mu \mathrm{L}$ \\
\hline
\end{tabular}

5. Assemble the digestion-ligation reaction as described below:

\begin{tabular}{|l|l|}
\hline B/c vector $(50 \mathrm{ng})$ & $\mathrm{X} \mu \mathrm{L}$ \\
\hline Diluted annealed oligonucleotides & $1 \mu \mathrm{L}$ \\
\hline $10 \times$ T4 DNA ligase buffer & $1 \mu \mathrm{L}$ \\
\hline T4 DNA ligase $(5 \mathrm{U} / \mu \mathrm{L})$ & $1 \mu \mathrm{L}$ \\
\hline BsaI $(10 \mathrm{U} / \mu \mathrm{L})$ & $1 \mu \mathrm{L}$ \\
\hline Sterile $\mathrm{H}_{2} 0$ & to $10 \mu \mathrm{L}$ \\
\hline
\end{tabular}

Use the amiRNA B/c vector of your choice (Fig. 3a and Fig. 4a). 
6. Mix the reactions by pipetting, and incubate for $5 \mathrm{~min}$ at $37^{\circ} \mathrm{C}$.

7. Transform 1-5 $\mu \mathrm{L}$ of the digestion-ligation into a $c c d \mathrm{~B}$-sensitive Escherichia coli strain (see Note 16).

8. Pick two colonies per construct, grow in $4 \mathrm{~mL}$ of liquid $\mathrm{LB}$ with kanamycin and purify plasmids with a miniprep kit.

9. Sequence two clones per construct with appropriate oligonucleotides: M13-F and M13-R for $p E N T R$-based vectors, attB1 and attB2 for $p M D C 32 B-, p M D C 123 S B-, p F K 210 B-$ or pH7WG2B-based vectors.

10. Transform $0.5 \mu 1$ of the purified plasmid into A. tumefaciens GV3101. Plate $1 / 10$ of the culture in a LB agar plate including rifampicin and kanamycin.

11. Incubate the plate at $28^{\circ} \mathrm{C}$ during $48 \mathrm{~h}$.

12. Store the plate with grown colonies at $4^{\circ} \mathrm{C}$ until use.

\subsection{Generation of Syn-tasiRNA Constructs}

\subsubsection{Syn-tasiRNA Design}

P-SAMS syn-tasiRNA Designer web app [9] is used to design syn-tasiRNAs with identical features than designed amiRNAs, that is a 5'U nucleotide, a $\mathrm{C}$ in position 19 and with a mismatch with the target transcript at position 21 . The app designs one or more syn-tasiRNA (see Note 17), each of which may target one or multiple genes in Arabidopsis thaliana and other closely related species that express the miR173 trigger (see Note 18). The app outputs the sequence of the syn-tasiRNA(s) together with the sequence of the two oligonucleotides required for cloning the syn-tasiRNA(s) in $\mathrm{B} / \mathrm{c}$ vectors containing the $A$. thaliana TASlc precursor (Fig. 5a) (see Note 4). The protocol described next is intended for the design of syntasiRNA(s) targeting endogenous plant transcripts. The possibility of designing syn-tasiRNAs to target exogenous transcripts is also discussed. 
1. Go to P-SAMS website (http://p-sams.carringtonlab.org/).

2. Click the "P-SAMS syn-tasiRNA Designer" application button to enter the syn-tasiRNA Designer tool (http://p-sams.carringtonlab.org/syntasi/designer).

3. To start the design process, click "Get Started" and then "Design syn-tasiRNAs" (see Note 19).

4. Select the transcriptome of the species of interest and click "Yes" (see Notes $\mathbf{8}$ and 20).

5. Click "Option 1" if you have the gene ID(s) of the target transcripts (see Note 8).

6. Enter one or more sets of target gene ID(s) (see Note 21). Each Gene set can contain one or more target gene ID(s). Click "Next" (see Note 18).

7. Click "Yes" to have the results automatically filtered based on target specificity (see Note 10).

8. Click "Submit" to submit the job (see Note 11).

9. Select "Click to see Results" to display results.

10. Navigate through the "Detailed Results" tab to display the list of "Optimal Results" and "Suboptimal Results" for each Gene set. Each result contains the syn-tasiRNA sequence and a summary of the target prediction analysis (score, target coordinates, target sequence, syn-tasiRNA/target base pairing and target description).

11. Click the "Build construct" tab to build the syn-tasiRNA construct.

12. Select the desired syn-tasiRNA by clicking and holding the corresponding box.

13. Drag it to the desired D3-D6 position on the bottom row, and drop it (see Note 22).

14. Repeat this process until all D3-D6 positions are filled.

15. The sequences of each of the selected syn-tasiRNAs and of the two oligonucleotides needed to clone the syn-tasiRNA cassette in a $T A S 1$-B/c-based vector (Fig. 5a) are then shown. Click "Download" to download this information. 


\subsubsection{Syn-tasiRNA Cloning}

Follow the steps described in 3.1.2 using the syn-tasiRNA vector of your choice (Fig. 5a). A diagram of the main steps for the high-thoughput cloning of amiRNA sequences in plant B/c vectors is shown in Fig. 5b.

\section{Notes}

1. Prepare $1 \mathrm{~mL}$ aliquots of Oligo Annealing buffer and store at $-20^{\circ} \mathrm{C}$ until use.

2. Order "B/c" vectors at Addgene website (http://www.addgene.org/).

3. Visit http://p-sams.carringtonlab.org/faq for video tutorials on how to design amiRNAs with P-SAMS amiRNA Designer app.

4. If you want to clone your artificial sRNA into a totally different vector system, you can still use P-SAMS to design the artificial sRNA and disregard the information related to the two oligonucleotides compatible with $\mathrm{B} / \mathrm{c}$ vectors.

5. If you already have an amiRNA guide sequence and you just want to generate oligos compatible with cloning in a B/c vector, click "Generate oligos" instead. Next Click "Monocot" or "Eudicot" if you want to use your amiRNA(s) in a monocot or in a eudicot species, respectively. Enter or paste an amiRNA sequence, and click the "+" button for entering additional amiRNA sequences. Click submit to get results.

6. If a eudicot or a monocot species is selected, at the end of the design process P-SAMS will output oligos for cloning into compatible amiRNA eudicot (AthMIR390a-based) or monocot (OsaMIR390-based) B/c vectors, respectively (Fig. 3a and Fig. 4a).

7. A large menu of plant species is available, but if your species of interest is missing then request its addition at administrator@carringtonlab.org.

8. Click “Option 2" to target an unannotated or exogenous transcript (e.g. a viral RNA). Enter or paste the FASTA sequence(s) of target transcript(s) and click "Next". Click "Yes" to 
have the results automatically filtered based on target specificity, and "Submit" to submit the job. Select "Click to see Results" to display the results.

9. Click the "+" button for entering additional target gene IDs to target multiple genes.

10. Clicking "Yes" will activate a target prediction module. Results that have predicted undesired targets will be discarded. Click "No" to deactivate the target prediction module.

11. Jobs generally take only a few minutes to finish. Leave open the browser's tab to access results when ready.

12. Due to the relatively long runtime requirements of the target prediction module, P-SAMS web is set up to return only up to three optimal results. Use P-SAMS script "psams.pl" (https://github.com/carringtonlab/p-sams) in the command line with the "- u" option activated to obtain all possible optimal results.

13. If you prefer to clone your artificial sRNA in a different vector system, it is recommended to clone the artificial sRNA insert in a $p E N T R$-based $\mathrm{B} / \mathrm{c}$ vector, and then transfer it to the vector of your choice (e.g. by LR recombination if the vector is GATEWAY compatible).

14. Alternatively, the annealing reaction can be done in a water bath or thermoblock by heating during $5 \mathrm{~min}$ at $94^{\circ} \mathrm{C}$ and then turning off the apparatus. Let the reaction to cool down until it reaches room temperature.

15. Do not store the diluted oligonucleotides.

16. The digestion-ligation reaction can be transferred to a spin column for nucleic acid purification. This optional step increases the number of colonies obtained after E. coli transformation.

17. Visit http://p-sams.carringtonlab.org/faq for video tutorials on how to design syntasiRNAs with P-SAMS syn-tasiRNA Designer app. 
18. Note that only Arabidopsis thaliana and close related species (e.g. Camelina sativa) produce miR173 required for triggering syn-tasiRNA biogenesis from TAS1c precursors. Therefore, if a different species is selected you should co-express miR173 to produce syntasiRNAs.

19. If you already have syn-tasiRNA guide sequences and you just want to generate oligos compatible with cloning in a B/c syn-tasiRNA vector, click "Generate oligos" instead. Next enter or paste an syn-tasiRNA sequence, and click the "+" button for entering additional syn-tasiRNA sequences. Click submit to get results.

20. When entering more than one gene ID, please separate gene IDs with a comma as shown in the examples. A syn-tasiRNA sequence will be designed for each Gene set, and the syntasiRNA for each set can be combined in any order after the design step is completed.

21. The number of syn-tasiRNAs to multiplex in your construct is decided at this point. We have successfully tested multiplexing up to four syn-tasiRNAs in a single construct [10]. Importantly, the farther from the miR173 target site the syn-tasiRNA is cloned, the less it accumulates in vivo suggesting that syn-tasiRNAs located too far away from miR173 target site may be poorly expressed and, consequently, possibly inactive. Thus, we decided to limit to four the number of syn-tasiRNAs that can be multiplexed in a single construct using P-SAMS syn-tasiRNA Designer.

22. Available positions are in light grey, and unavailable positions are in dark grey.

\section{Ackowledgements}

This work was supported by grants BIO2014-54269-R and BIO2017-83184-R from the Ministerio de Economía, Industria y Competitividad (Spain). Alberto Carbonell was the recipient of an Individual Fellowship from the European Union's Horizon 2020 research and innovation programme under the Marie Sklodowska Curie grant agreement No. 655841. 


\section{References}

1. Carbonell A (2017) Artificial small RNA-based strategies for effective and specific gene silencing in plants. In: Dalmay T (ed) Plant Gene Silencing: Mechanisms and Applications. CABI Publishing, pp 110-127

2. Schwab R, Ossowski S, Riester M, Warthmann N, Weigel D (2006) Highly specific gene silencing by artificial microRNAs in Arabidopsis. Plant Cell 18 (5):1121-1133. doi:10.1105/tpc. 105.039834

3. Montgomery TA, Howell MD, Cuperus JT, Li D, Hansen JE, Alexander AL, Chapman EJ, Fahlgren N, Allen E, Carrington JC (2008) Specificity of ARGONAUTE7-miR390 interaction and dual functionality in TAS3 trans-acting siRNA formation. Cell 133 (1):128141. doi:10.1016/j.cell.2008.02.033

4. Montgomery TA, Yoo SJ, Fahlgren N, Gilbert SD, Howell MD, Sullivan CM, Alexander A, Nguyen G, Allen E, Ahn JH, Carrington JC (2008) AGO1-miR173 complex initiates phased siRNA formation in plants. Proc Natl Acad Sci U S A 105 (51):20055-20062. doi:10.1073/pnas.0810241105

5. de la Luz Gutierrez-Nava M, Aukerman MJ, Sakai H, Tingey SV, Williams RW (2008) Artificial trans-acting siRNAs confer consistent and effective gene silencing. Plant Physiol 147 (2):543-551. doi:10.1104/pp.108.118307

6. Carbonell A (2017) Plant ARGONAUTEs: Features, Functions, and Unknowns. Methods Mol Biol 1640:1-21. doi:10.1007/978-1-4939-7165-7_1

7. Carbonell A, Takeda A, Fahlgren N, Johnson SC, Cuperus JT, Carrington JC (2014) New generation of artificial MicroRNA and synthetic trans-acting small interfering RNA vectors for efficient gene silencing in Arabidopsis. Plant Physiol 165 (1):15-29. doi:10.1104/pp.113.234989 
8. Carbonell A, Fahlgren N, Mitchell S, Cox KL, Jr., Reilly KC, Mockler TC, Carrington JC (2015) Highly specific gene silencing in a monocot species by artificial microRNAs derived from chimeric miRNA precursors. Plant J 82 (6):1061-1075. doi:10.1111/tpj.12835 9. Fahlgren N, Hill ST, Carrington JC, Carbonell A (2016) P-SAMS: a web site for plant artificial microRNA and synthetic trans-acting small interfering RNA design. Bioinformatics 32 (1):157-158. doi:10.1093/bioinformatics/btv534

10. Carbonell A, Daros JA (2017) Artificial microRNAs and synthetic trans-acting small interfering RNAs interfere with viroid infection. Mol Plant Pathol 18 (5):746-753. doi:10.1111/mpp.12529

11. Carbonell A, Carrington JC, Daros JA (2016) Fast-forward generation of effective artificial small RNAs for enhanced antiviral defense in plants. RNA Dis 3 (1):e 1130. doi:10.14800/rd.1130

12. Carbonell A, Daros JA (2018) Design, Synthesis and Functional Analysis of Highly Specific Artificial Small RNAs With Antiviral Activity in Plants. Methods Mol Biol (in press) 


\section{Figure Captions}

Fig. 1. Artificial sRNA pathways in plants. (a) The amiRNA pathway. An amiRNA transgene, containing a plant miRNA precursor in which the original miRNA/miRNA* sequences have been substituted by the amiRNA/amiRNA* sequences, is introduced into plants to express an amiRNA primary transcript processed into an amiRNA foldback. A rationale amiRNA design requires that the amiRNA foldback preserves the original secondary structure of the endogenous precursor. DCL1 further processes the amiRNA foldback to produce the amiRNA duplex methylated by HEN1. The 5'U amiRNA strand is incorporated usually into AGO1 to silence highly complementary transcripts by direct slicing or by repressing their translation. (b) The syn-tasiRNA pathway. A syn-tasiRNA transgene, containing a plant $T A S$ precursor in which a subset of the original tasiRNA sequences has been substituted by several syn-tasiRNA sequences in tandem, is introduced into plants to express a syn-tasiRNA primary transcript. An endogenous miRNA cleaves this primary transcript, a process that triggers the recruitment of RDR6 complexes to synthesize a dsRNA from one of the cleavage products. DCL4 processes the dsRNA into phased tasiRNA duplexes in $21 \mathrm{nt}$ register with the miRNA cleavage site. SyntasiRNA guide strands with a 5'U are incorporated into AGO1 to direct specific silencing of sequence unrelated target transcripts at one or multiple sites. Reproduced from Carbonell 2017 [1] with permission from CAB International (Wallingford, UK).

Fig. 2. Flowchart of the main steps from artificial sRNA design to plant transformation using P-SAMS and B/c cloning vectors. Reproduced from Carbonell 2017 [1] with permission from CAB International (Wallingford, UK).

Fig.3. High-throughput cloning of amiRNAs in AtMIR390a-B/c-based vectors for use in eudicots. (a) Diagram of an AtMIR390a-B/c Gateway-compatible entry vector ( $p E N T R$ AtMIR390a-B/c) (top), and of $A t M I R 390 a-B / c$-based binary vectors for expression of amiRNAs in eudicots (pMDC32B-AtMIR390a-B/c, pMDC123SB-AtMIR390a-B/c and 
pFK210B-AtMIR390a-B/c, bottom). RB: right border; 35S: Cauliflower mosaic virus promoter; BsaI: BsaI recognition site, $c c d \mathrm{~B}$ : gene encoding the $c c d \mathrm{~B}$ toxin; LB: left border; attL1 and attL2: gateway recombination sites. $\mathrm{Kan}^{R}$ : kanamycin resistance gene; $H y g^{R}$ : hygromycin resistance gene; $\operatorname{Basta}^{R}$ : glufosinate resistance gene; Spec ${ }^{R}$ : spectinomycin resistance gene. Undesired $B s a \mathrm{I}$ sites removed from the plasmid are crossed out. (b) Diagram of the main steps for high-throughput cloning of amiRNAs in AtMIR390a-B/c-based vectors containing a modified version of AtMIR390a precursor that includes a $c c d \mathrm{~B}$ cassette flanked by two inverted $B s a \mathrm{I}$ sites. The amiRNA insert obtained after annealing the two partially complementary and overlapping oligonucleotides has $5^{\prime}$ overhangs compatible with those resulting from the $B$ saI digestion of the $\mathrm{B} / \mathrm{c}$ vector where it is inserted directionally. The DNA fragments corresponding to the AtMIR390a precursor, the amiRNA and the amiRNA* are in black, blue and green respectively. (a) is adapted from Carbonell et al. 2014 [7], and (b) is reproduced from Carbonell 2017 [1] with permission from CAB International (Wallingford, UK).

Fig. 4. High-throughput cloning of amiRNAs in $O S M I R 390-B / c$-based vectors for use in monocots. (a) Diagram of an OsMIR390-B/c Gateway-compatible entry vector ( $p E N T R$ OsMIR390-B/c) (top), and of OsMIR390-B/c-based binary vectors for expression of amiRNAs in mnocots (pMDC32B-OsMIR390a-B/c, pMDC123SB-OsMIR390a-B/c and $p H 7 W G 2 B-$ OsMIR390-B/c, bottom). OsUB: Ubiquitin promoter from rice. Other details are the same as in Figure 4a. (b) Diagram of the main steps for high-throughput cloning of amiRNAs in OsMIR390-B/c-based vectors containing a modified version of OsMIR390 precursor that includes a $c c d \mathrm{~B}$ cassette flanked by two inverted $B s a \mathrm{I}$ sites. The DNA fragments corresponding to the OsMIR390 precursor, the amiRNA and the amiRNA* are in grey, blue and green respectively. Other details are the same as in Figure 3b. Adapted from Carbonell et al. 2014 $[7]$. 
Fig. 5. High-throughput cloning of syn-tasiRNAs in $A t T A S 1 c-B / c$-based vectors for use Arabidopsis and close relatives. (a) Diagram of an $A t T A S 1 c-B / c$ Gateway-compatible entry vector ( $p E N T R$-AtTAS1c-B/c) (top), and of $A t T A S 1 c-B / c$-based binary vectors for expression of syn-tasiRNAs (pMDC32B-AtTAS1c-B/c, pMDC123SB-AtTAS1c-B/c and $p F K 210 B-$ AtTAS1c-B/c, bottom). Other details are as in Fig. 3a. (b) Diagram of the main steps for highthroughput cloning of syn-tasiRNAs in $A t T A S 1 c-B / c$-based vectors containing a modified version of AtTASlc precursor that includes a $c c d \mathrm{~B}$ cassette flanked by two inverted BsaI sites. The syn-tasiRNA insert obtained after annealing the two partially complementary and overlapping oligonucleotides has $5^{\prime}$ overhangs compatible with those resulting from the $B s a I$ digestion of the $\mathrm{B} / \mathrm{c}$ vector where it is inserted directionally. The DNA fragments corresponding to the AtTASIc precursor, the syn-tasiRNA-1 and the syn-tasiRNA-2 are in black, blue and green respectively. Adapted from Carbonell et al. 2014 [7]. 
a amiRNA pathway

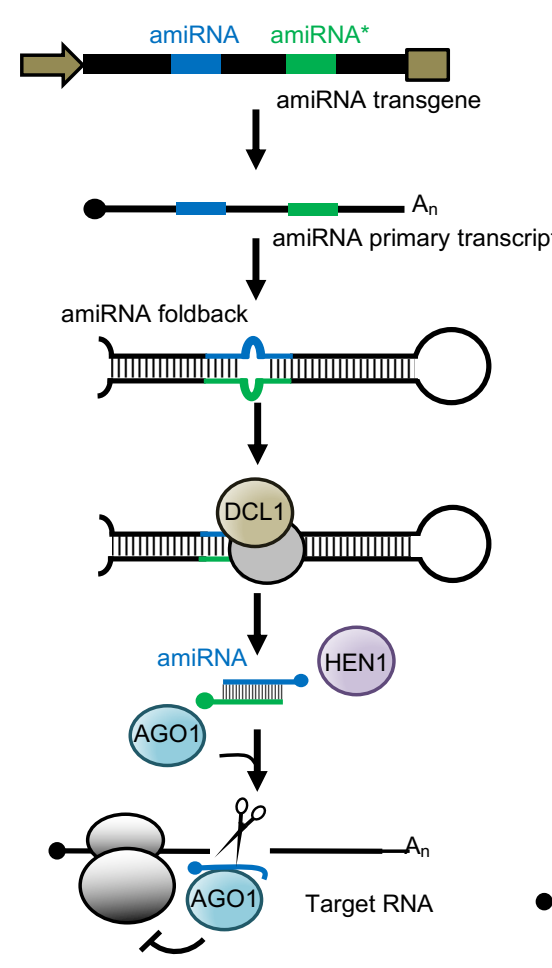

b syn-tasiRNA pathway
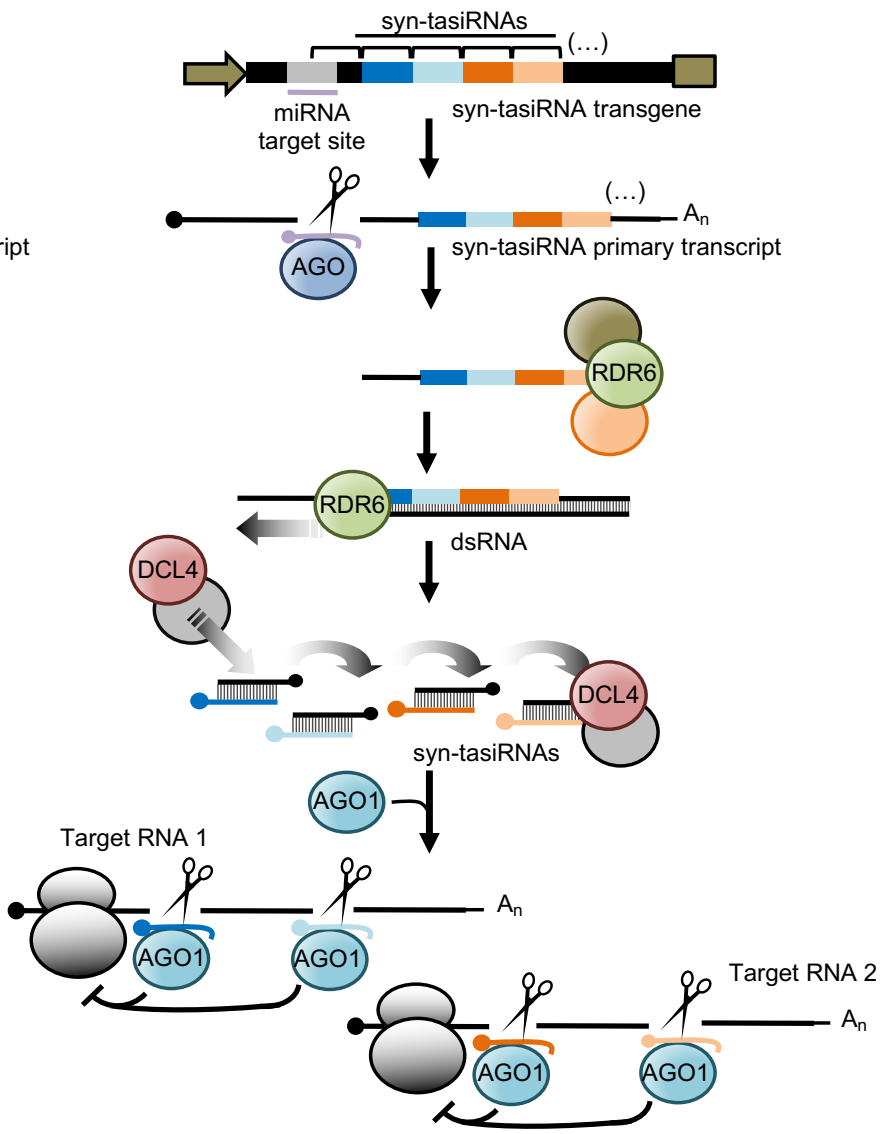


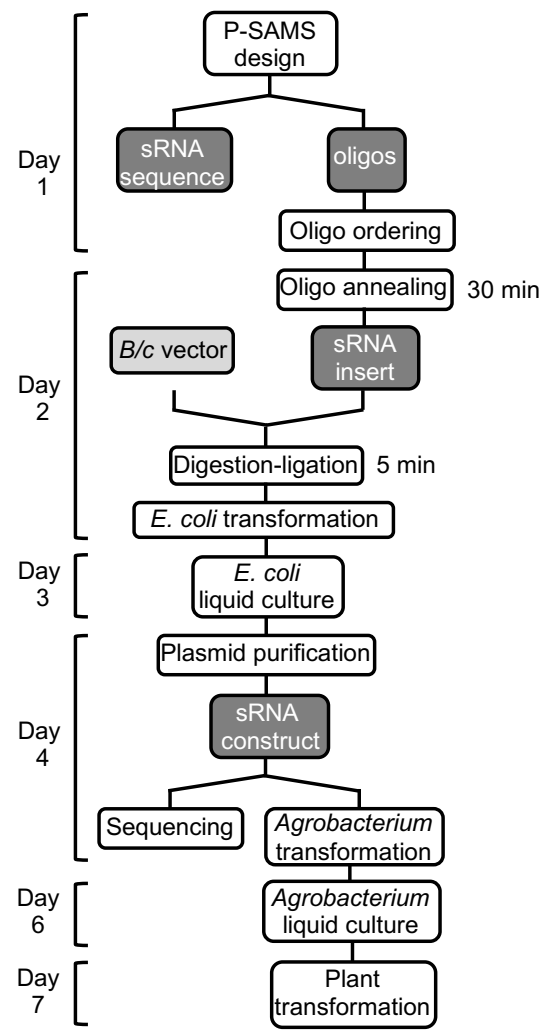

FIG. 2 
a b

Gateway-compatible AtMIR390a-B/c entry vector

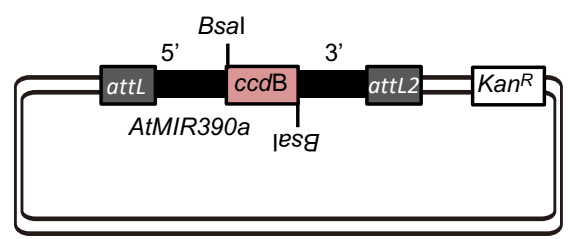

pENTR-AtMIR390a-B/c

(Addgene \#51778)

Plant binary AtMIR390a-B/c vectors

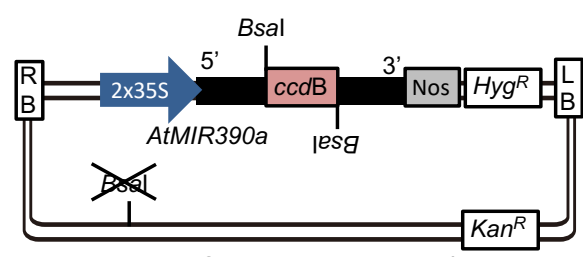

pMDC32B-AtMIR390a-B/c

(Addgene \#51776)

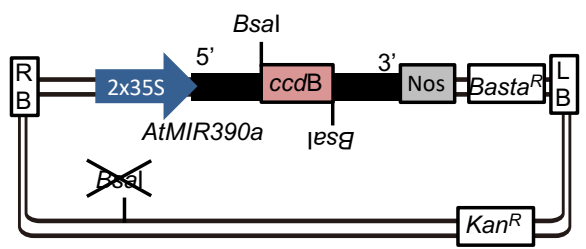

pMDC123SB-AtMIR390a-B/c

(Addgene \#51775)

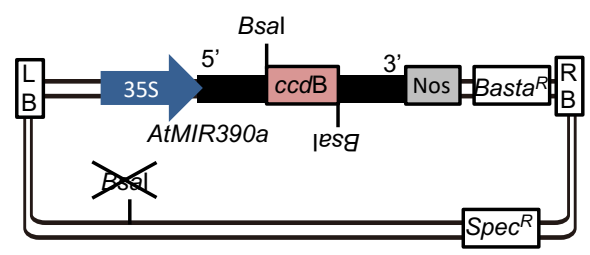

pFK210B-AtMIR390a-B/c

(Addgene \#51777)
AtMIR390a-B/c vector

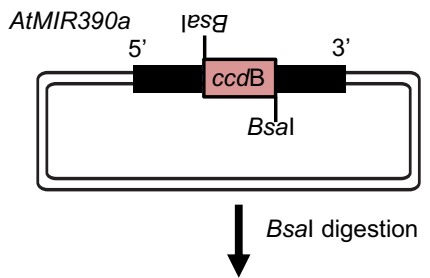

CATT

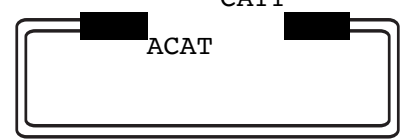

$\downarrow$ Ligation

AtMIR390a-based amiRNA construct amiRNA amiRNA*

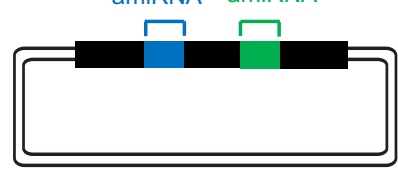


a b

Gateway-compatible OSMIR390-B/c entry vector

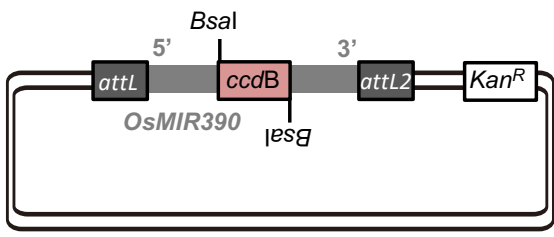

pENTR-OsMIR390-B/c

(Addgene \#61468)

Plant binary OsMIR390-B/c vectors

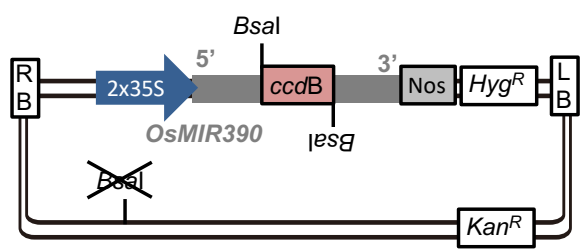

pMDC32B-OsMIR390-B/c

(Addgene \#61467)

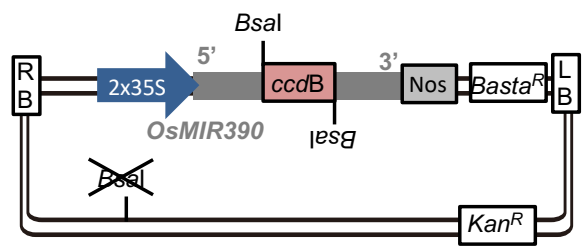

pMDC123SB-OsMIR390-B/C

(Addgene \#61466)

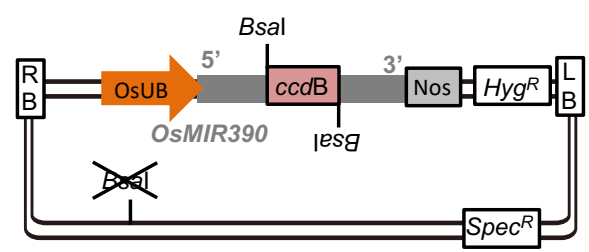

pH7WG2B-OsMIR390-B/c

(Addgene \#61465)

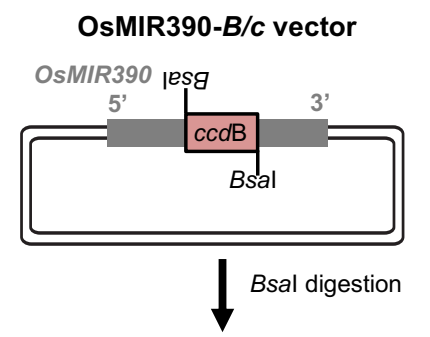

Overlapping oligonucleotides

amiRNA amiRNA*

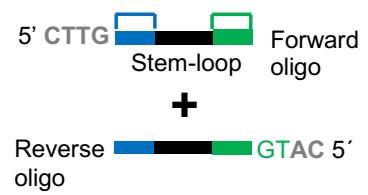

oligo

Oligonucleotide annealing

\section{amiRNA insert}

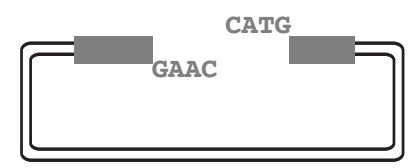

amiRNA amiRNA*

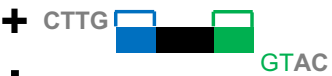

Ligation

OsMIR390-based amiRNA construct

amiRNA amiRNA*

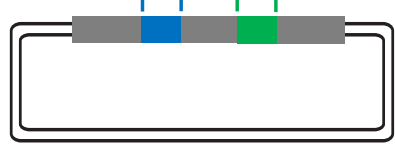


a

Gateway-compatible AtTAS1c-B/c entry vector

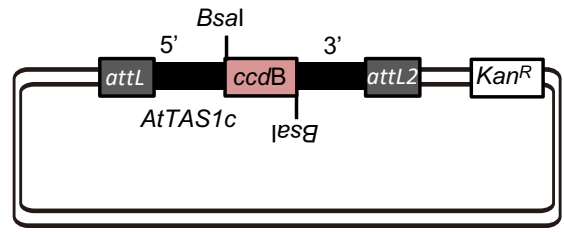

pENTR-TAS1c-B/c

(Addgene \#51774)

Plant binary AtTAS1c-B/c vectors
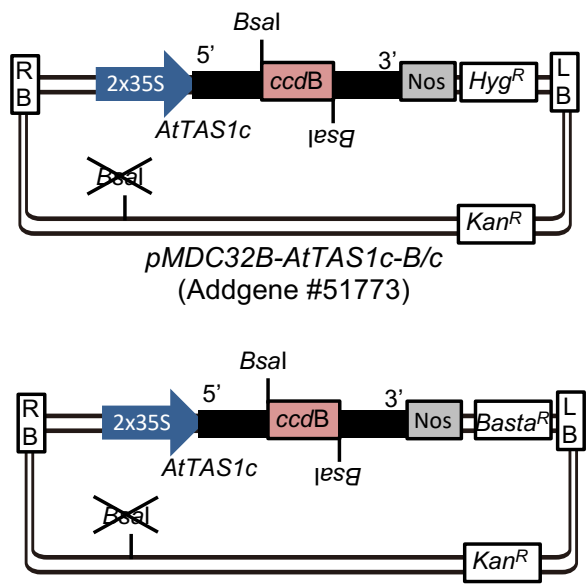

pMDC123SB-AtTAS1C-B/C

(Addgene \#51772) b

AtTAS1c-B/c vector

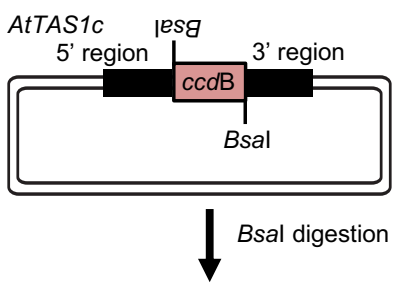

syn-tasiRNA-1 syn-tasiRNA-2 5' ATTA $\square$ Forward

Reverse CTTG 5' oligo

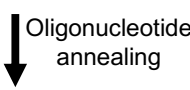

syn-tasiRNA insert

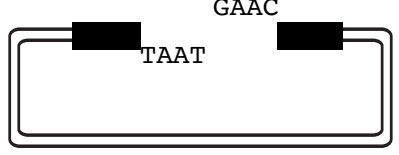

syn-tasiRNA-1 syn-tasiRNA-2

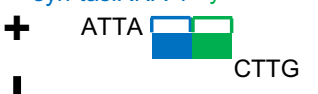

Ligation

AtTAS1c-based syn-tasiRNA construct

syn-tasiRNA-1 syn-tasiRNA-2

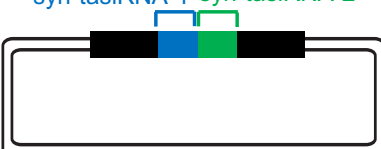

